\title{
DEVELOPMENT OF PC BASED TEST FACILITY FOR SENSOR CHARACTERIZATION
}

\author{
Ajay Kumar Keshari, J. Prabhakar Rao \\ Chemical Facilities Division, Chemistry Group, Indira Gandhi Centre for Atomic \\ Research, Kalpakkam - 603102, Tamil Nadu, India \\ ajayanu@igcar.gov.in, jprao@igcar.gov.in
}

\begin{abstract}
A PC based test facility has been developed for rapid and in-depth study of the characteristics of sensor materials that are being developed in Chemistry Group of IGCAR. We have developed an operational interface module for the commercially available Source Measurement Unit. User programmable parameters like set voltage/current, sample rate are provided in the front panel. The resulting Current/voltage is acquired and logged.

As one of the important applications of the system, it was used to study the transport number of an ionic species in a compound. This can be found out by passing a known current or voltage for a specified duration and measure the output voltage or current respectively. From the initial and final current exhibited by the sample, the transport number can be deduced. The transport number of the Lithium ion in Lithium Zirconium Phosphate has been studied and found the results which are meeting with requirements.
\end{abstract}

\section{Keywords}

Source Measure Unit, Gas Sensor, LabVIEW, GPIB, Transport Number

\section{INTRODUCTION}

The study of electrical characteristics of a compound material is necessary while developing sensors. It is preferred to conduct the experiment in a programmed and automatic way so that the results would be accurate. It is also required to make the experimental procedure automatic to get the results faster. This paper focuses on the development of a user friendly experimental facility and also a typical application of the system to study the transport number of an ionic species in a compound.

The development comprises of a commercially available versatile Source Measurement Unit (SMU), fully programmable instrument, capable of sourcing voltage/current and measuring current/voltage simultaneously. It has the GPIB interface for external control. Basically the SMU is being operated manually. We have developed the interfacing module for the SMU and user friendly operational routine to conduct the experiment. The Graphical User Interface (GUI) program is developed on a versatile LabVIEW 8.0 to interface the GPIB of the SMU. LabVIEW is a graphical development environment for creating flexible and scalable design, control, and test applications rapidly. LabVIEW has mainly two panels, Front Panel and Block Diagram. Front Panel is the user interface screen where the command buttons, data display gadgets are present. Block Diagram is the place where LabVIEW coding is done graphically .The routine is provided with operational features to generate the required excitation signal to the sensor for the user

DOI : 10.14810/elelij.2015.4103 
defined period and to measure the resulting signal. The signal is also logged at a user defined sample rate and plotted in a graphical display. The user can optimize the values of source voltage/current, time, number of counts etc., and get the results to predict the behavior of sensor. The instrument can conduct the experiment automatically once it is programmed. The transport number of Lithium ion in Lithium Zirconium Phosphate sensor has measured and also studied the characterization of sensor. This setup has also been used to study the VI characteristics of semiconductor devices.

\section{DEVELOPMENT AND IMPLEMENTATION}

The sensor is connected to Source Measurement Unit (SMU). SMU is connected to Personal Computer via GPIB interface. The Block diagram of overall setup is shown in Figure.1

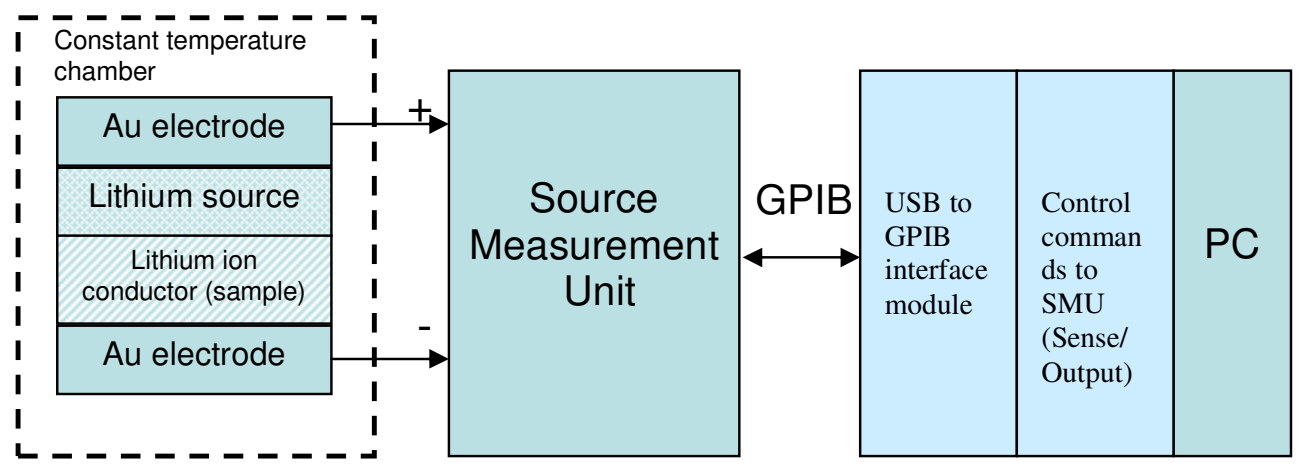

Figure 1. Block Diagram of complete Setup

\subsection{Methology}

SMU is capable of automatically source and measure voltage and current simultaneously using GPIB interface commands. The output of SMU is connected to the sample setup which may be in a confined environment. The SMU sources the proper level of voltage to the sensor and measures the current across the sensor. User optimizes the proper level of source voltage, time etc., across sensor and measure the corresponding current .By measuring the initial and final current user can calculate the transport number and study the behavior of sensor.

\subsection{Software}

A Graphical User Interface program has the provision to set the mode and level of the voltage/current for counting. Two stages of counting are provided, fast and then slow counting. There is a provision for saving the acquired data in a file, displaying the graph and in table form where user can see the behavior of sensor. The data saved in the file is useful for post analysis of sensor behavior and for calculating the transport number.

\subsection{Program Flow}

The sequential flow of the task is shown in the flow chart as shown in Figure 2. The implementation of the task is done in LabVIEW Block Diagram as shown in Figure 3, and the user Front Panel is shown in Figure 4. User can program two sets of counts, each set containing the number of samplings and delay time between the samplings. The voltage input is also set by 
the user. The data is saved in a file as per user input. All the above data is taken from user and the program initializes the file configuration. Then the program initializes the GPIB communication to Source Measurement Unit (SMU).

The trigger for SMU is set and made the SMU in listen mode. The read configuration is implemented and then acquired the data from SMU. The acquired data is saved in the file entered by the user. This file can be directly imported to origin or excel for post analysis. But the program displays the data in the form of a graph so that user can get the idea of the trend profile at any instance. The saved file can be used for the accurate results and in depth analysis.

\subsection{Transport Number}

Transport number is described as a fraction of the total current carried by an ion. It is the ratio of the current carried by a given ionic species through a cross section of an electrolytic solution to the total current passing through the cross section. It is a characteristic dependent on the mobility of all the ions in the electrolytic solution, concentrations of the ions, and on the temperature of the solution.

The sample for which the transfer number is required is sandwiched between two blocking electrodes and a DC voltage is applied across the electrodes. When the circuit is closed, the instantaneous current (Ii) gives a measure of total conductivity (electronic and ionic). The time variation of the current through the sample gives an idea about the polarization at the electrodeelectrolyte interface. The final stabilized current $\left.\left(\mathrm{I}_{\mathrm{f}}\right)\right)$ is the electronic conductivity. The ionic transfer number can be determined using relation

Tion $=\left(\mathrm{Ii}-\mathrm{I}_{\mathrm{f}}\right) / \mathrm{Ii}$ 


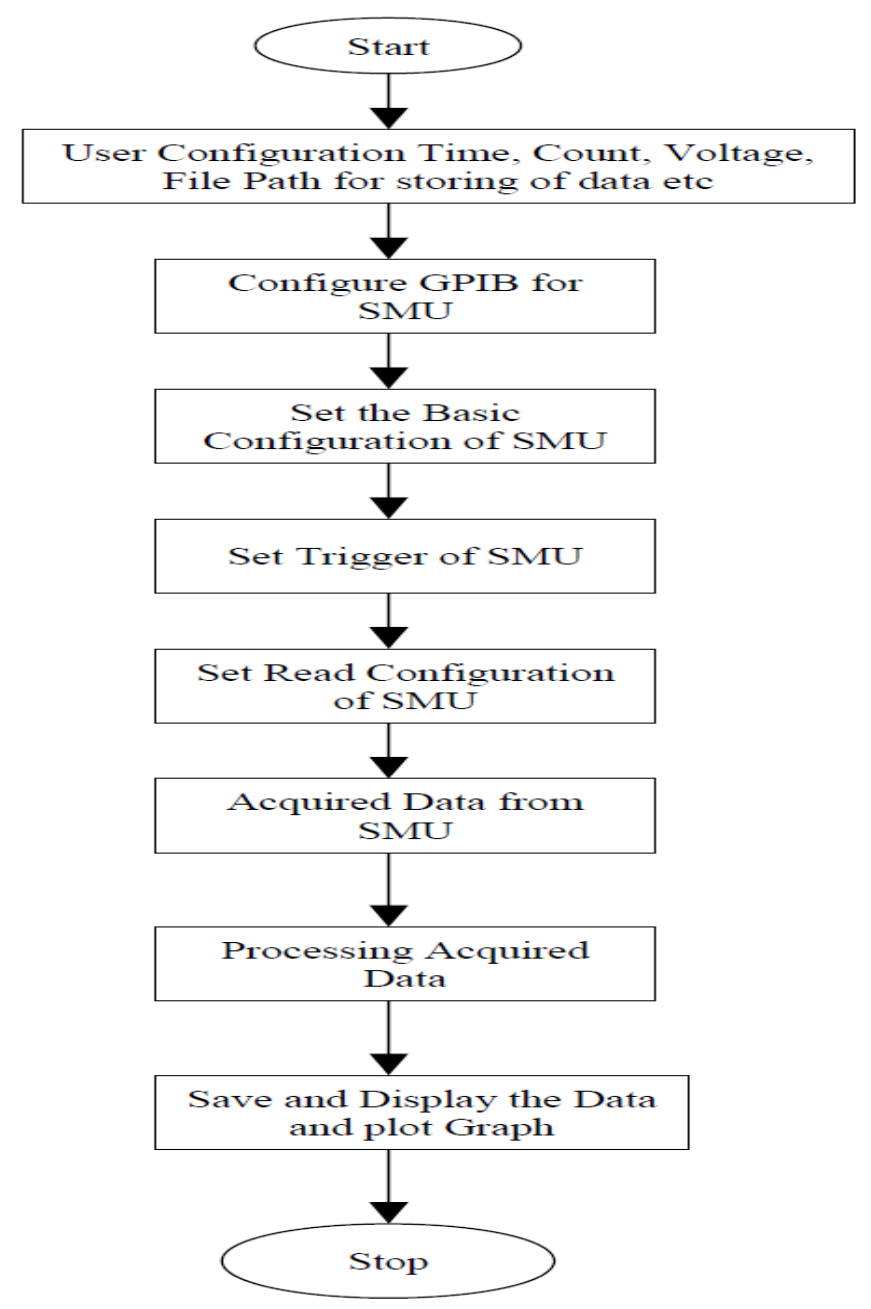

Figure 2. Flow chart of the program

\subsection{Experiment}

The sample Lithium Zirconium Phosphate in the form of solid electrolyte has sandwiched between electrodes in glass. The GUI allows the user to set faster sampling rate for rapid portion of the sensor response profile and slower sampling rate for settling portion of the sensor response. For example, the user can collect a fixed number of samplings (counter number 1) of signal at every $\mathrm{ms}$; and after the period the user would collect second set of fixed number samplings (counter number 2) of data points at every 2 seconds. In both cases sampling interval is programmable. This feature allows the user to choose between the conflicting parameters like speed and memory requirement. The measured data is stored in a file. The same data is also shown as graph. The graph is having zoom facilities like horizontal zoom, vertical zoom, and window zoom etc.

Here in Chemistry Group of IGCAR, one of the experiments conducted utilizes this setup is to find out the transport number of an ionic species in a compound [1]. This can be found out by 
Electrical and Electronics Engineering: An International Journal (ELELIJ) Vol 4, No 1, February 2015

passing a constant voltage for a specified duration and measure the resulting current characteristic of the sensor material. The data collection is done in appropriate with the sampling rate programmed by the user. From the initial and final current exhibited by the sample, the transport number can be deduced.

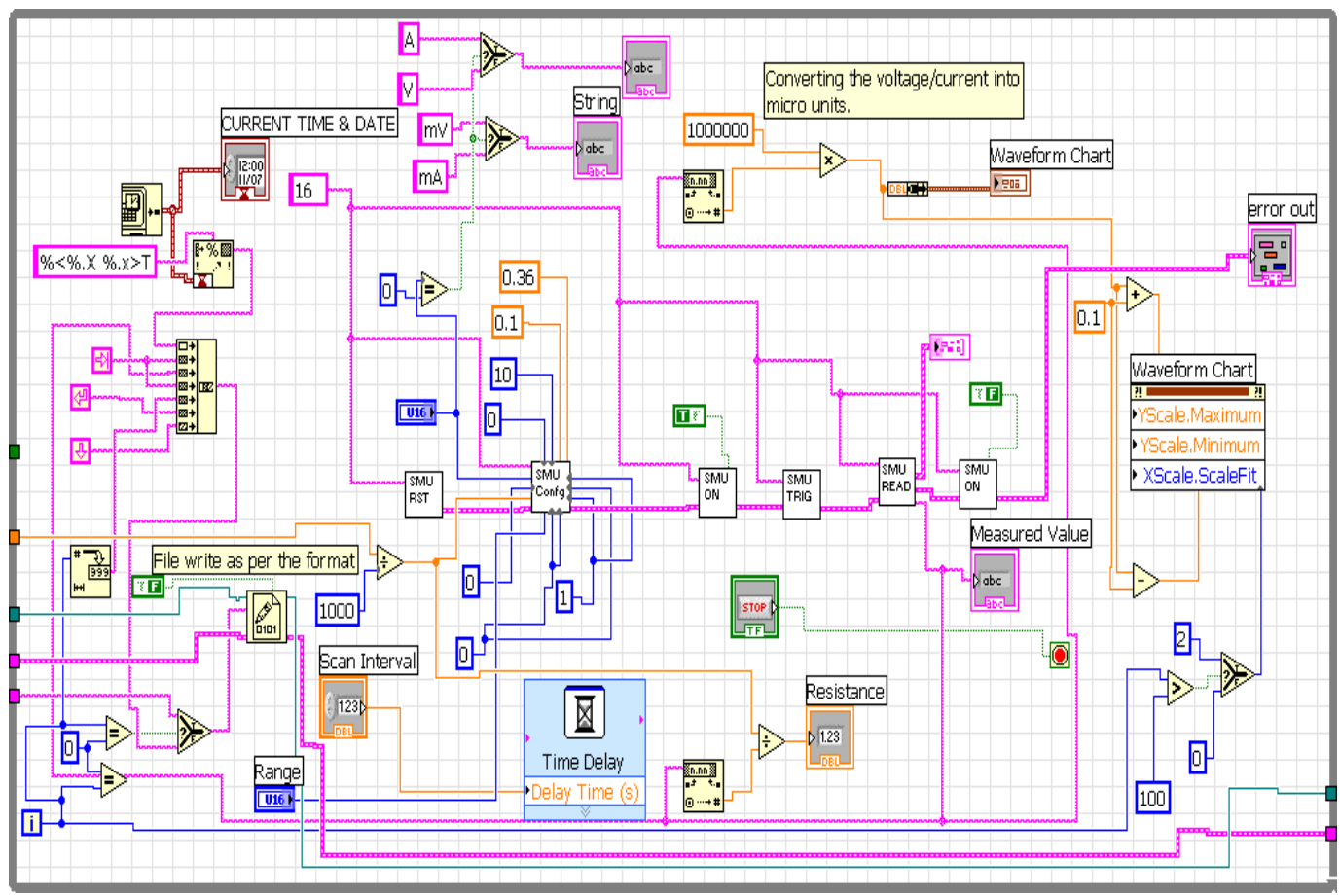

Figure 3. Block Diagram of the Program

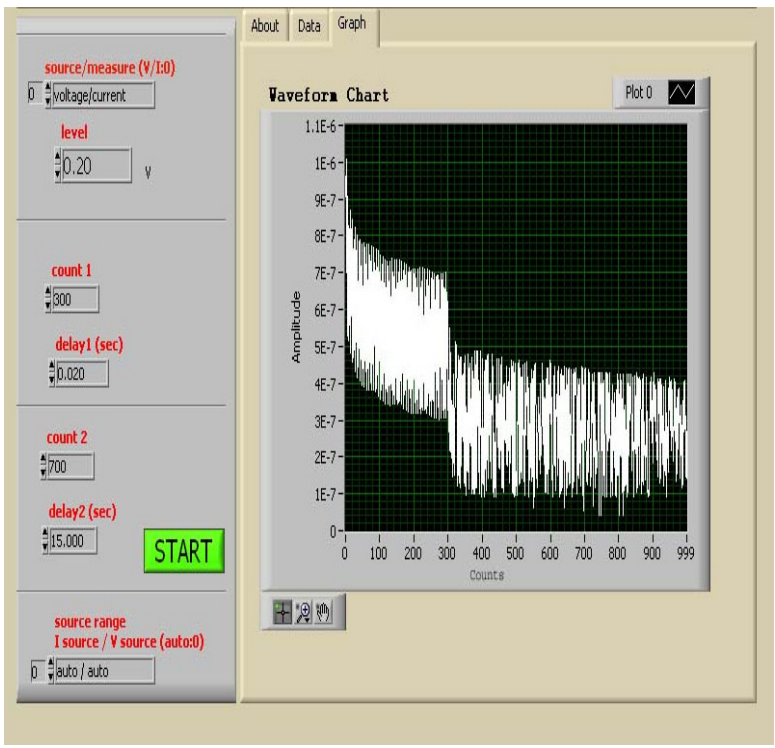




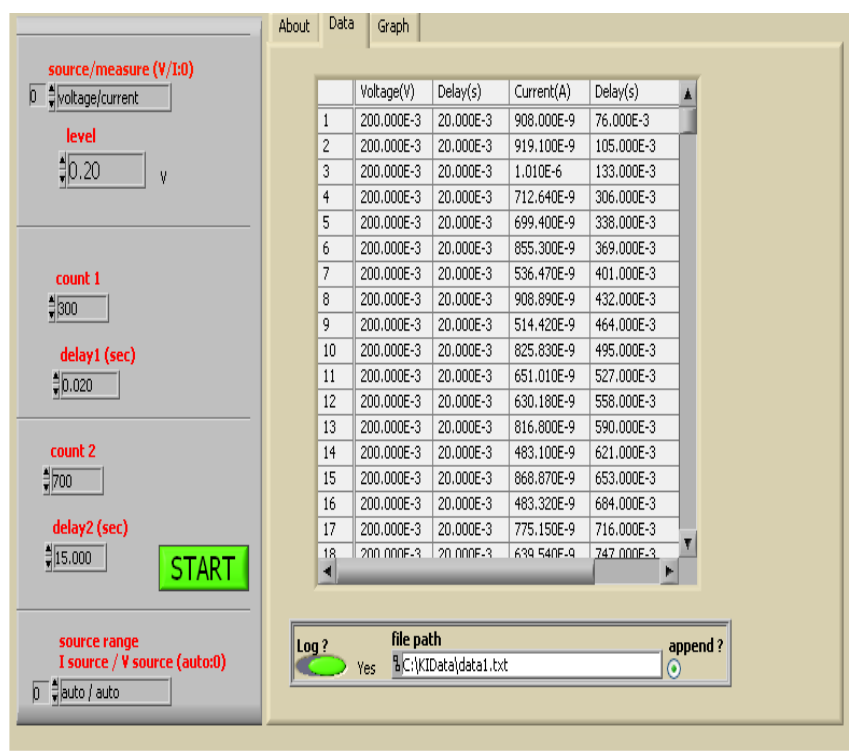

Figure 4. Front Panel of the Program

\section{RESULTS}

In a typical experiment conducted in our laboratory, the transport number of Lithium in a compound Lithium Zirconium Phosphate was found. The sample in the form of solid electrolyte sandwiched between $\mathrm{Au}$ on one side and $\mathrm{Au}, \mathrm{Li}_{2} \mathrm{CO}_{3}+\mathrm{Ag}$ on other side housed in a glass chamber. Two leads are taken from both the sides of the sample. The sample was kept in a chamber maintained at $400^{\circ} \mathrm{C}$ temperature. Typically $200 \mathrm{mV}$ was applied across the sample using the SMU and the current was measured as a function of time. The initial current $I_{i}$ i.e., the total current contributed by ionic (Lithium) and electronic species and the final current at later time $\mathrm{I}_{\mathrm{f}}$ i.e., current due to the electronic species alone were obtained from these experiments. The resulting current would be an initial fast decaying microampere current and then a slow stable nanoampere current. Configuring the first sampling rate counter for small time and the second counter for large time does the precise measurement. It was observed that the initial current $I_{i}$ was large and the final current $\mathrm{I}_{\mathrm{f}}$ was smaller. The transport number was deduced by using the equation

$\mathrm{T}_{\mathrm{Li}}=\mathrm{I}_{\mathrm{i}}-\mathrm{I}_{\mathrm{f}} / \mathrm{I}_{\mathrm{i}}$

The transport number of the Lithium ion in Lithium Zirconium Phosphate was found as 0.8837 and 0.8804 for different samples which agrees with the expected values; indicating that the major contribution to conductivity is due to Lithium ions only. Figure 5. depicts a couple of responses obtained from this setup. 

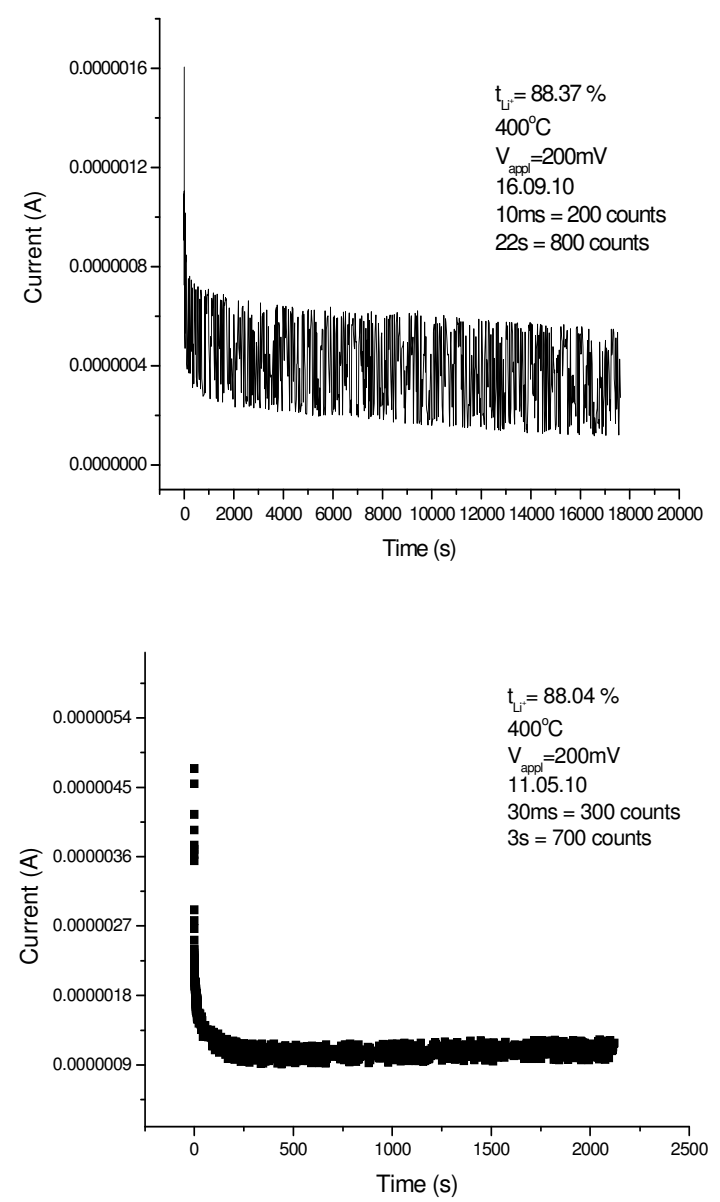

Figure 5 Graphs Showing Current as a Function of Time

\section{CONCLUSIONS}

This set up is very useful for conducting experiments related to sensor material characterization studies. It provides more accurate and fast measurement facility. It makes easy to export data in graphical as well as text format. It has flexibility of configuring the sample rate counters making the experiments customized. This is also used to find out the V-I characteristics of the diode. This can also be used in finding the characteristics of the semiconductor devices as well.

\section{Acknowledgements}

The authors are very grateful to Shri. A. Sreeramamurthy, MCD, Chemistry Group, IGCAR for his help in conducting the experiment and getting the results.

\section{References}

[1] S.S. Sunu, V. Jayaraman, E. Prabhu, K.I. Gnanasekar and T. Gnanasekaran, " $\mathrm{Ag}_{6} \mathrm{Mo}_{10} \mathrm{O}_{33}-\mathrm{A}$ New Silver Ion Conducting Ammonia Sensor Material", $2^{\text {nd }}$ International Conference on Ionic Devices, Anna University, India, Nov. 28-30, 2003. 
Electrical and Electronics Engineering: An International Journal (ELELIJ) Vol 4, No 1, February 2015

[2] http://www.ni.com

[3] Reference Manual, LabVIEW 8.0

\section{Authors}

Mr. Ajay Kumar Keshari is an electronics engineer (Scientific Officer /D) and working in instrumentation for Chemistry Group. His expertise is in control electronics, embedded systems and software development using LabVIEW and Visual Basic. The hardware design is done using microcontrollers and Programmable System on Chip (PSoC).

Mr. J. Prabhakar Rao is an electronics engineer (Scientific Officer/E) working in Chemistry Group having vast experience in instrumentation and automation. His expertise is in control electronics, embedded systems and software development. He has done automation of many legacy instruments using microcontrollers, Programmable System on Chip (PSoC) using software like LabVIEW \& Visual Basic.
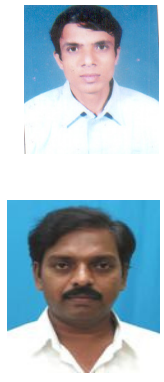